\title{
New hope for Nutlin-3a therapy for pulmonary arterial hypertension
}

\author{
Jutaro Fukumoto, Richard Lockey and Narasaiah Kolliputi * \\ Division of Allergy and Immunology, Department of Internal Medicine, Morsani College of Medicine, University of South Florida, Tampa, FL, USA \\ *Correspondence: nkollipu@health.usf.edu \\ Edited by: \\ Mario Malerba, University of Brescia, Italy
}

\section{A commentary on}

Activation of lung p53 by Nutlin-3a prevents and reverses experimental pulmonary hypertension

by Mouraret, N., et al. (2013) Circulation 127, 1664-1676. doi: 10.1161/circulationaha.113.002434

Pulmonary Arterial Hypertension (PH) is a chronic, progressive, and fatal lung disease characterized by narrowing of pulmonary arteries associated with muscular thickening and intimal proliferation, known as remodeling. If untreated, the 5-year survival rate of $\mathrm{PH}$ is 34\% (McLaughlin et al., 2002). Unfortunately, there is no systematic strategy established to effectively manage this devastating disease. Currently, the best curative option for $\mathrm{PH}$ is lung transplantation. It, however, is applicable for only a few patients mainly due to the lack of suitable donors. Moreover, even if lung transplantation is successfully performed, the patients have to take immunosuppressant for the rest of their life. Administration of long-term immunosuppressant has been associated with high incidence of side effects including severe and sometimes fatal infections.

The FDA-approved medications for $\mathrm{PH}$ fall into three categories: (i) prostacyclin derivative, which is a very potent vasodilator and also a inhibitor of pulmonary artery smooth muscle cell (PASMC) proliferation, (ii) endothelin-1 receptor antagonist, which counteracts vasoconstriction and PASMC proliferation, and (iii) phosphodiesterase-5 inhibitor, which causes pulmonary vasodilatation and inhibition of PASMC proliferation. These vasodilators have certainly achieved significant advances in the management of $\mathrm{PH}$ during the last decade. However, because of their short half-lifes and narrow therapeutic ranges, close monitoring and/or follow-up is required to avoid excessive side effects involved in vasodilation. Some $\mathrm{PH}$ patients with severe conditions even require continuous intravenous or subcutaneous administration of these drugs, which can be a big clinical challenge for both patients and health care providers. To get a better way in managing $\mathrm{PH}$ conditions and in maintaining longterm patient adherence, a new drug that specifically and strongly blocks PASMC proliferation, a key pathological event in $\mathrm{PH}$, is desired.

In the last decade, a good line of evidence has revealed that there is a variety of similarities existent between PH and cancer (McMurtry et al., 2005; Dromparis et al., 2010; Aljubran et al., 2012). Two representatives of them are uncontrolled cellular proliferation and resistance to apoptosis. In the recent issue of Circulation, Mouraret et al. present an elegant study on the effects of Nutlin-3a on PH (Mouraret et al., 2013). Nutlin$3 \mathrm{a}$, which is a cis-imidazoline analog, was originally developed as an anti-cancer drug. It stabilizes p53 by inhibiting the binding of MDM2 (murine double minute 2), a p53-specific E3 ubiquitin ligase that promotes p53 degradation (Vassilev et al., 2004). p53, a representative tumor suppressor protein, plays a critical role in tumor suppression through several different mechanisms primarily involving the DNA repair process. Recently, it has been reported that $\mathrm{p} 53$ deletion deteriorates hypoxia-induced $\mathrm{PH}$ in mice (Mizuno et al., 2011). Consistent with this report, Mouraret et al. revealed that a p53 stabilizer Nutlin-3a reversed $\mathrm{PH}$ in three distinct experimental mouse models with only limited side effects noted in control mice without $\mathrm{PH}$. Moreover, they confirmed that Nutlin-3a requires p53 and p21 expression to work as an antiPH drug showing that Nutlin-3a exerts no effects on hypoxia-exposed $\mathrm{p} 53^{-/-}$ and $\mathrm{p} 21^{-/-}$mice. Intriguingly, the paper also shows that Nutlin-3a treatment of cultured human PASMCs results in cell growth arrest but not apoptosis, which is consistent with other reports using fibroblasts from mice and humans (Efeyan et al., 2007; Kumamoto et al., 2008). Therefore, it appears that Nutlin-3a has a totally different mechanism of action from currently available drugs and that it is not directly associated with relaxation of smooth muscle and vasodilation of the pulmonary arteries, which lessens the concern about the side effects related to vasodilation. If Nutlin-3a works well for treatment of $\mathrm{PH}$ patients and it becomes an approved therapeutic application, $\mathrm{PH}$ patients would be able to have more therapeutic options including combination therapies, which ultimately would lead to decreased number of $\mathrm{PH}$ patients with advanced stages. The benefit of expanded therapeutic options could have an impact in decreasing the number of $\mathrm{PH}$ patients waiting for lung transplantation. On the other hand, one minor drawback of using Nutlin-3a as a potential $\mathrm{PH}$ treatment is its slow clinical effect on the patients with severe $\mathrm{PH}$ due to its lack of direct vasodilatory effect. However, in spite of the above mentioned disadvantage, this new drug still has an enormous therapeutic potential for treatment of $\mathrm{PH}$. The day may come before long when Nutlin3a will come out as a blessing for $\mathrm{PH}$ patients.

\section{ACKNOWLEDGMENTS}

Authors thank Dr. Diane S. Allen-Gipson for critical reading this commentary. Narasaiah Kolliputi was funded by the American Heart Association National Scientist Development Grant 
09SDG2260957 and National Institutes of Health R01 HL105932 and the Joy McCann Culverhouse Endowment to the Division of Allergy and Immunology.

\section{REFERENCES}

Aljubran, S. A., Cox, R. Jr., Tamarapu Parthasarathy, P., Kollongod Ramanathan, G., Rajanbabu, V., Bao, H., et al. (2012). Enhancer of zeste homolog 2 induces pulmonary artery smooth muscle cell proliferation. PLoS ONE 7:e37712. doi: 10.1371/ journal.pone.0037712

Dromparis, P., Sutendra, G., and Michelakis, E. D. (2010). The role of mitochondria in pulmonary vascular remodeling. J. Mol. Med. (Berl.) 88, 1003-1010. doi: 10.1007/s00109-010-0670-x

Efeyan, A., Ortega-Molina, A., Velasco-Miguel, S., Herranz, D., Vassilev, L. T., and Serrano, M. (2007). Induction of p53-dependent senescence by the mdm2 antagonist nutlin-3a in mouse cells of fibroblast origin. Cancer Res. 67, 7350-7357. doi: 10.1158/0008-5472.CAN-07-0200

Kumamoto, K., Spillare, E. A., Fujita, K., Horikawa, I., Yamashita, T., Appella, E., et al. (2008). Nutlin$3 \mathrm{a}$ activates $\mathrm{p} 53$ to both down-regulate inhibitor of growth 2 and up-regulate mir-34a, mir-34b, and mir-34c expression, and induce senescence. Cancer Res. 68, 3193-3203. doi: 10.1158/0008-5472.CAN07-2780

McLaughlin, V. V., Shillington, A., and Rich, S. (2002). Survival in primary pulmonary hypertension: the impact of epoprostenol therapy. Circulation 106, 1477-1482. doi: 10.1161/01.CIR.0000029100.82385.58

McMurtry, M. S., Archer, S. L., Altieri, D. C., Bonnet, S., Haromy, A., Harry, G., et al. (2005). Gene therapy targeting survivin selectively induces pulmonary vascular apoptosis and reverses pulmonary arterial hypertension. J. Clin. Invest. 115, 1479-1491. doi: 10.1172/ JCI23203

Mizuno, S., Bogaard, H. J., Kraskauskas, D., Alhussaini, A., Gomez-Arroyo, J., Voelkel, N. F., et al. (2011). P53 gene deficiency promotes hypoxia-induced pulmonary hypertension and vascular remodeling in mice. Am. J. Physiol. Lung Cell. Mol. Physiol. 300, L753-L761. doi: 10.1152/ajplung. 00286.2010

Mouraret, N., Marcos, E., Abid, S., Gary-Bobo, G., Saker, M., Houssaini, A., et al. (2013).
Activation of lung p53 by nutlin-3a prevents and reverses experimental pulmonary hypertension. Circulation 127, 1664-1676. doi: 10.1161/CIRCULATIONAHA.113.002434

Vassilev, L. T., Vu, B. T., Graves, B., Carvajal, D., Podlaski, F., Filipovic, Z., et al. (2004). In vivo activation of the p53 pathway by small-molecule antagonists of $\mathrm{mdm} 2$. Science 303, 844-848. doi: 10.1126/science. 1092472

Received: 06 June 2013; accepted: 20 June 2013; published online: 10 July 2013.

Citation: Fukumoto J, Lockey R and Kolliputi N (2013) New hope for Nutlin-3a therapy for pulmonary arterial hypertension. Front. Pharmacol. 4:87. doi: 10.3389/ fphar.2013.00087

This article was submitted to Frontiers in Respiratory Pharmacology, a specialty of Frontiers in Pharmacology. Copyright (c) 2013 Fukumoto, Lockey and Kolliputi. This is an open-access article distributed under the terms of the Creative Commons Attribution License, which permits use, distribution and reproduction in other forums, provided the original authors and source are credited and subject to any copyright notices concerning any third-party graphics etc. 